\title{
I. Durabilidad del hormigón
}

\author{
Resistencia química de los cementos
}

PABlo GARCIA DE PAREDES GAIBROIS, Ledo. en Química

Por fortuna, actualmente, cuantos intervienen en el proyecto, la elaboración y el curado de las obras en hormigón han adquirido plena conciencia de las condiciones y cuidados necesarios para asegurar una "vida útil" al hormigón que permita llegar al término de la misma sin menoscabo de sus propiedades físico-mecánicas; es decir, que sea "durable".

Uno de los factores que condicionan en forma influyente la durabilidad del hormigón frente a las características de un entorno dado, es la resistencia química que la pasta cementicia endurecida ofrezca a los diversos agentes que existen en los ambientes, y especialmente al medio físico-químico que se halla en el interior de los poros y canalilios del hormigón y que está condicionado por la composición del medio ambiente exterior.

Por desgracia no se dispone, todavía, de un modo de proceder o método que permita apreciar la resistencia química de la pasta en un plazo de tiempo congruente con el ritmo de las construcciones y que posea una fiabilidad suficiente.

Las investigaciones realizadas con el fin de normalizar un método rápido y suficientemente exacto se han referido, con especial énfasis, al aspecto pacticular de la resistencia a los efectos idestructores de los medios líquidos que contienen azufre en la forma de sulfatos.

La naturaleza de los suelos ibéricos así como el desarrollo de sus costas colocan a los sulfatos como los factores más destructores del hormigón en España. La experiencia diaria en la "España yesífera" ha puesto de manifiesto que al yeso le acompaña frecuentemente el ion magnesio. En cuanto al agua del mar de acción más compleja, puede incluirse siquiera parcialmente, en el grupo 2. (Sulfatos) de la RILEM que, en cuanto a frecuencia, ocupa en España parejo lugar a la corrosión de las armaduras. Ambos "modos" se hallan estrechamente relacionados con las tensiones internas y la cristalización de sales, ya que, según $\mathrm{O}$. Valenta, la destrucción del hormigón por iones $\mathrm{SO}_{4}{ }^{2-}, \mathrm{Cl}^{-}, \mathrm{Ca}^{2+}$, $\mathrm{Mg}^{2+}, \mathrm{Na}^{+}, \mathrm{NH}_{4}{ }^{+}$, etc. resulta de un, complejo y muy diverso, conjunto de procesos que se conjugan en gran número de posibles combinaciones entre las propiedades de los componentes del hormigón y la simultaneidad o secuencia de los variados factores perjudiciales o beneficiosos.

Es innegable que la durabilidad de un hormigón depende en grado máximo del valor que alcancen, en el tiempo, sus cualidades de compacidad y permeabilidad. Pero estas características están en gran parte condicionadas por el tipo de conglomerante utilizado. 
La forma más satisfactoria de ensayar la "sulfatodurabilidad" de los hormigones es la observación del cambio que sus propiedades físicas experimentan cuando se colocan piezas de hormigón en contacto del o de los agresivos en condiciones reales: Pilotes hincados en terrenos o en puertos de mar; carreteras experimentales, redes de tuberías, etc. La duración que estas observaciones requieren y la imposibilidad de asegurar una identidad en las circunstancias entre ensayos paralelos o sucesivos, merma mucho el valor de este modo de proceder.

Por esos motivos se impone el empleo de métodos que suministren, en un plazo relativamente corto, la información deseada sin mengua notable de la veracidad del diagnóstico; es decir, hay que utilizar métodos acelerados.

O. Valenta distingue en los métodos acelerados, los que se pueden considerar como "científicos" y aquéllos que se pueden designar como "técnicos" debido a su carácter pragmático así como a carecer, frecuentemente, de una base suficientemente conocida.

Todo método "científico" debe basarse en el conocimiento, más completo y reciente, de los procesos que ocurren en las estructuras durante su permanencia en los ambientes agresivos o favorables. Por ello estos métodos aparecen en continua evolución. Los métodos "técnicos", que en realidad son en su mayor número aplicaciones más o menos parciales de los anteriores, deben reunir las siguientes cualidades:

Veracidad: Para ello el proceso de alteración debe ser igual al mecanismo real que ocurre en las estructuras o, por lo menos, se debe conocer previamente la diferencia entre ambos, así como el sentido que los componentes y cualidades de la estructura imprimen o pueden imprimir a los resultados del ensayo, especialmente en cuanto a la velocidad de alteración.

Reproducibilidad: Es una condición ideal o límite, pero no puede eliminarse la necesidad de conocer, con la mayor certeza que sea posible, en qué medida se pueden obtener resultados concordantes al repetir el ensayo conservando la máxima identidad posible en las condiciones operativas.

Sencillez: Cuantas menos variables intervengan en el ensayo, tanto más fácil será la deducción lógicamente correcta de conclusiones.

Aceleración: Esta cualidad impone la máxima prudencia al interpretar los resultados.

El empleo de un análisis estadístico es casi imprescindible a causa de la complejidad inevitable de influencias que tanto merman la exactitud.

Es también conveniente no conformarse con un solo tipo de ensayos o sólo con trabajar con pasta, o mortero. El diagnóstico responderá tanto mejor a una visión real del problema cuanto más amplio sea el panorama abarcado. Distintos serán los ensayos de rutina de los que habrían de utilizarse en la elaboración de un proyecto o informe; no digamos para sentar una doctrina.

En primer plano surge la elección de los parámetros cuya variación de magnitud o, mejor aún, su velocidad de variación (no se puede olvidar que la durabilidad es propiedad temporal, J. Calleja) se utilizará como expresión de la cualidad ensayada.

Para R. H. Bogue existen cinco "pistas" de observación: El aspecto exterior. El valor de las resistencias mecánicas. Las dimensiones de las probetas. El módulo de elasticidad. Las composiciones químicas, tanto de las probetas como del medio agresor.

I. Biczok, se fija principalmente en la forma adoptada para acelerar el proceso que, según él, puede conseguirse por: Incremento de la superficie de contacto entre la pasta conglomerante endurecida y el agente agresor. Aumento de la presión que, al cristalizar, produzcan los compuestos originados al actuar el agresivo. Arrastre por disolución o des- 
gaste de los componentes de la pasta, al incrementar la penetración o la filtración del agresivo. Aumento de la concentración del agresivo. Elevación de la temperatura del sistema agresivo-probeta. Tanto Bogue como Biczok se complementan en sus hipótesis.

Las divergencias que suelen aparecer en las calificaciones de los conglomerantes establecidas a partir de los resultados obtenidos aplicando métodos acelerados, generalmente se deben a dos motivos: Aplicación del método fuera del entorno de validez propio del mismo. Utilización de un método cuyo proceso físico-químico difiera mucho o no represente los fenómenos reales del caso estudiado.

Nadu Mihai presentó al II Coloquio Internacional sobre la Durabilidad del Hormigón, celebrado en Praga el año 1969, la siguiente clasificación de los métodos utilizables para el diagnóstico de la durabilidad:

\section{METODOS BASADOS EN EL CONTACTO DE MORTEROS Y HORMIGONES CON DISOLUCIONES AGRESIVAS QUE DIFIEREN DE LAS CONDICIONES REALES}

\subsection{Aumento de la concentración del ion $\mathrm{SO}_{4}{ }^{2-}$}

Koch, A. y Steinegger, H. Zement Kalk Gips 1960-VII-317 a 321.

Resistencias a flexotracción.

Lea, F. M. The Chemistry of Cementand Concrete.

Análisis químico del agresivo.

Nadu, M. Standardizarea. 1966-XII págs. 637/42.

Aumento de la concentración de $\mathrm{SO}_{4}{ }^{2-}$.

Norma rumana STAS E-2633-67.

Resistencias a tracción.

Thorwaldson, T. IV Simposium on Chemistry of Cement. London 1952.

Aumento de la concentración de $\mathrm{SO}_{4}{ }^{2-}$. 
1.2. Modificación de la composición del agente agresivo. Adición de iones $\mathbf{M g}^{2+}, \mathbf{N H}_{4}^{+}$, $\mathrm{Cl}^{-}, \mathbf{y}$ otros

Bogue, R. H.; Taylor, W. Lerch, W. Ind. Eng. Chem. 1934, pág. 1.048.

Análisis químico del agresivo.

Hägermann, Y. Zement 1957, n. ${ }^{\circ} 14$.

Análisis químico del agresivo.

\subsection{Aumento de la temperatura}

Biczok, I. Concrete Corrosion, Concrete protection, 1964.

\section{METODOS BASAdOS EN EL AUMENTO DE LA SUPERFICIE DE CONTACTO ENTRE EL CONGLOMERANTE ENDURECIDO Y EL AGENTE AGRESIVO}

2.1. Por pulverización probable del conglomerante endurecido

2.1.1. Introducción, en débil proporción, del agente agresivo

Steopoe, A. Ciment si Beton. 1935. XI y XII, pág. 321 (Rumania).

Análisis de los cambios experimentados por la pasta endurecida.

2.1.2. Mezcla con sulfato cálcico pulverizado y preparación con esta mezcla, de probetas que se curan en cámara húmeda

Le Chatelier Anstett. Rev. Mat. 1935. XI, n. ${ }^{\circ} 314$.

Blondiau, h. Rev. Mat. 1961, III, n. ${ }^{\circ} 546$.

Aspecto de las probetas. Cambios dimensionales. 
Jasper, M. J. M. Rev. Mat. 1968. VI. n. 633.

Aspectos de las probetas. Cambios dimensionales.

Norma holandesa NEN 1591.

Norma rumana STAS-E 2633-67.

Cambios dimensionales.

Simpliceau V. Hidrotechnie. 1968. VIII. Rumania.

Cambios dimensionales.

2.1.3. Igual procedimiento que en 2.1.2., pero sustituyendo el $\mathrm{CaSO}_{4}$ parcial o totalmente por otros sulfatos $(\mathrm{Mg}, \mathrm{Na}, \mathrm{K})$

Jasper, M. J. M. Rev. Mat. 1968. VI, n. 633.

Cambios dimensionales.

2.2. Endurecimiento de un mortero normalizado a cuyo conglomerante se le ha incrementado la cantidad de yeso

Norma A.S.T.M. C 452-64 T.

Cambios de longitud.

2.3. Filtración bajo presión del agente agresivo a través de probetas de pasta, de mortero o de hormigón

Orth, J. Bull. Rilem 1968, X. pág. 35.

Aspecto externo de la probeta. 
2.4. Utilización de probetas de pequeñas dimensiones pero con gran superficie exterior

Koch, A. y Steinegger, H. loc. cit.

Resistencia a flexotración.

Merriman, T. Fort-Peck, Dam. Specifications 1933.

García de Paredes y Gaibrois, P.

RILEM. Symposium Durability of Concrete. 1969. Final report. A. 41.

Fernández-Peña Secades, O.

RILEM Symposium. Durability of Concrete. Final report. C-103.

Norma checoslovaca CSR. CSN 773-56.

Norma rumana STAS E 2633-67.

Norma rusa GOST 4798-49.

Cambios de longitud.

3. METODOS FISICOS BASADOS EN LA SUSTITUCION DE LOS FENOMENOS ESPECIFICOS DE EXPANSION PROVOCADOS POR LA CORROSION, POR LA PRESION DE CRISTALIZACION DE SALES CONTENIDAS EN LOS AGRESIVOS

Norma Alemana DIN 52.111.

4. METODOS QUIMICOS BASADOS EN LA SUSTITUCION TOTAL DE LOS COMPLEJOS PROCESOS QUIMICOS, FISICOS Y MECANICOS POR REACCIONES QUIMICAS EN SISTEMA HETEROGENEO Y SENCILLO

4.1. Sedimentación en copos de los complejos sulfato-alumínicos formados en dispersiones acuosas, diluidas, de conglomerantes

Paul, I. Proc. Assos Highway Officials 1936.

Análisis químico del filtrado o del sólido. 


\subsection{Solubilización de los conglomerantes en disoluciones de azúcar}

Merriman, T. Fort-Peck, Dam. Specifications 1933.

Análisis químico (Alcalimetría).

4.3. Solubilización de los sulfatos contenidos en el conglomerante que no han reaccionado, mediante la dispersión en agua de cal

Bogue, R. H. The Chemistry of Portland Cement. 1955.

Análisis químico del filtrado.

Consideramos de interés el conocimiento detallado de los diversos modos o métodos que los investigadores han seguido con la intención de conocer y, en la medida posible, predecir, la resistencia química que poseen los cementos hidráulicos frente a los agresivos más frecuentes, como son las aguas y los terrenos que contienen sulfatos.

Para ello iniciamos la publicación de los métodos a nuestro alcance exponiéndolos, en la medida posible, con un desarrollo en forma de norma. Esperamos excitar, de esta forma, el afán investigador de los interesados en este tema.

Confiamos poder, en el futuro, publicar la valiosa colaboración que nos sea remitida y cuyo interés, para una posible solicitud de normalización, es evidente. 


\section{Ensayo de la durabilidad frente a los sulfatos}

D. G. MILLER y C. C. SNYDER

La durabilidad se aprecia mediante cambios de longitud de los prismas de mortero mantenidos en contacto con disoluciones de sulfatos.

1) Materiales. Moldes: De acero duro capaces para obtener prismas de 2,5 $\times 2,5 \times 12,5$ $\mathrm{cm}$. Se pueden utilizar los moldes que se emplean en la preparación de los prismas cortos del ensayo en autoclave. Indices: De acero inoxidable. Se pueden utilizar los del ensayo de autoclave.

2) Arena: La silícica del tipo de la de Ottawa (20-30). Sulfatos: Los que se deseen ensayar serán de la calidad "Para análisis".

3) Proporciones: La mezcla será de 1:5 ponderal. La relación agua/cemento se calculará según la fórmula siguiente: (ASTM C 190-44).

$$
y=\frac{2}{3} \frac{P}{n+1}=K
$$

En la cual:

$y=$ Porcentaje de agua que requiere el mortero.

$P=$ Porcentaje de agua necesario para la pasta de consistencia normal del cemento.

$n=$ Número de partes de arena que corresponden a una de cemento, en peso.

$K=$ Una constante que para la arena de Ottawa (20-30) tiene el valor de 6,5.

Cada tongada de 6 prismas requiere $1.200 \mathrm{~g}$ de arena, $240 \mathrm{~g}$ de cemento y la cantidad de agua que el cálculo indique. En un cemento con $25,0 \%$ de agua para la consistencia normal, la cantidad calculada de agua para amasado será 133,6 $\mathrm{ml}$ (9,28\%).

4) Preparación de los moldes: Los moldes se recubrirán interiormente de una capa ligera de vaselina sólida (petroleum jelly) y se colocarán con todo cuidado los índices que no deberán mancharse de grasa (se utilizan moldes cortos del ensayo de estabilidad en autoclave). Los índices (de acero inoxidable) se rodean de pasta de cemento. Para ello se colocan en los extremos del molde unos trozos de cartón que se ha embebido en aceite mineral y dejado secar durante una noche, provistos de un orificio circular en su centro, de $8 \mathrm{~mm}$ de diámetro. Este cartón se ajusta bien al extremo del molde; para colocar la capa de pasta de cemento de $6 \mathrm{~mm}$ de espesor es muy útil servirse de una tira de hierro en uno de cuyos extremos se ha practicado una ranura en forma de U (para salvar el índice); el espesor de la chapa puede ser de $4 \mathrm{~mm}$. También se colocará transversalmente (como una tapadera) sobre el extremo una chapa de hierro rectangular de $6 \mathrm{~mm}$ de ancho, siempre que se quiera conocer cuánto falta aún para completar el espesor de pasta deseado.

5) Amasado del mortero: Debe realizarse según se indica en la sección 6 del método ASTM C 305.

6) Confección de los prismas: En primer lugar se colocarán las capas terminales de pasta de cemento que rodearán a los índices, según se ha indicado en 4). A continuación se colocará el mortero sobre los extremos del molde, aproximadamente $2 \mathrm{~cm}$ de espe- 
sor, que se presionará sobre la capa de pasta mediante seis golpes con el dedo índice derecho en la dirección del eje del molde mientras con el índice de la mano izquierda se sujeta el otro extremo del molde. Una vez colocadas estas capas de mortero adyacentes a las de pasta pura que rodean a los índices metálicos, se completa el contenido del molde en dos capas en forma similar a como se procede en el ensayo de constancia de volumen medida con autoclave. ASTM C 151.

Es de sumo interés que durante todas las operaciones de enmoldado, que pueden realizarse en 12 a 15 minutos, se mantenga el local de trabajo a una temperatura de $23^{\circ} \pm 2{ }^{\circ} \mathrm{C}$ y la humedad relativa oscile entre 60 y $65 \%$.

7) Curado de los prismas: Los prismas deberán permanecer en los moldes de 46 a 48 horas y. encerrados en la cámara húmeda.

Inmediatamente de la extracción de los prismas, se les marcará en forma que su identificación no deje lugar a dudas, se medirá su longitud con el auxilio del comparador y se anotará este dato inicial, y se les conservará sumergidos en agua potable durante 5 días.

Al completarse los 7 días de edad se repite la medición y anotación de los datos y se les pesa.

A partir de esta fecha se les introduce en las disoluciones agresivas, repitiéndose las pesadas y medidas cada 28 días.

Antes de cada medida del peso y de la longitud, los prismas se limpian ligeramente con un cepillo o pincel plano de $3 \mathrm{~cm}$ aproximadamente de ancho que se pasará cinco veces a lo largo de cada una de las caras de los prismas.

Al pesar los prismas, se les debe secar quitando el agua superficial con un paño o papel absorbente.

Los cambios de longitud se calculan como porcentajes referidos a la longitud efectiva (distancia entre los extremos interiores de los índices) de los prismas a la edad de 7 días. La relación entre el volumen de los prismas y el de la disolución de ataque en cada uno de los depósitos deberá mantenerse en el valor 1:4. Las concentraciones de las disoluciones se expresarán en molaridades.

Para conservar las disoluciones se utilizarán vasijas tapadas y se las conservará a $23^{\circ} \pm 3^{\circ} \mathrm{C}$. Las disoluciones se renovarán cada 7 días.

Los prismas se colocarán en los recipientes que contienen las disoluciones agresivas, en posición horizontal sobre enrejado de madera o de metal no atacable. La rejilla se mantendrá aproximadamente a $2,5 \mathrm{~cm}$ del fondo de la vasija y entre los prismas deberá haber una distancia mayor de $6 \mathrm{~mm}$ (1/4"). De cada amasada se deberán ensayar en cada disolución dos prismas, y el ensayo comprenderá no menos de seis barras preparadas en 3 días diferentes.

Las disoluciones agresivas serán:

- Sulfato sódico $0,15 \mathrm{M}(2,1 \%)$.

- Sulfato magnésico 0,15 M (1,8\%).

- Como testigo o ensayo en blanco: Agua potable.

8) Valoración de los resultados: Se considerará que un cemento no soporta satisfactoriamente el ensayo si el valor medio de la expansión de los seis prismas exceda a $0,02 \%$ en cada una de las dos disoluciones, si la variación de peso es mayor del $5 \%$ o si los prismas se han pandeado notablemente. 


\section{Ensayo de sulfato-durabilidad utilizado por el Buraeu of Reclamation}

Denver Colorado - U.S.A.

\section{F U N D A M E N T O}

La inalterabilidad del conglomerante se deduce de las variaciones que experimenta la longitud de las probetas cilíndricas, de mortero, curadas en cámara húmeda, sometidas después al ataque del sulfato sódico en disolución acuosa, y, por último, desecadas al aire.

\section{PROBETAS}

Cilindros de mortero. Altura: $15 \mathrm{~cm}$. Diámetro: $7,5 \mathrm{~cm}$.

- Tamaño máximo del árido: $17 \mathrm{~mm}$.

- Relación agua/cemento (en peso): 0,51.

- Escurrimiento: 7,50 cm.

- Aire ocluido: $5 \pm 1 \%$.

\section{APARATOS Y MOLDES}

a) Balanzas, pesas, probetas de vidrio graduadas, moldes y comparador, de acuerdo con las condiciones de "Aparatos para medir los cambios de volumen de la pasta de cemento, el mortero y el hormigón" (ASTIM C 490).

b) Mesa de sacudidas, de acuerdo con las condiciones de "Mesa de sacudidas para los ensayos de conglomerantes hidráulicos" (ASTM C 230).

c) Mezclador, cuenco y paleta, según las condiciones del "Método de la mezcla mecánica de las pastas y de los morteros plásticos de Cementos Hidráulicos" (ASTM C 30).

d) Palustre y pisón, de acuerdo con el "Método de ensayo de la resistencia a la compresión de los morteros hidráulicos" (ASTM C 109).

e) Moldes, de acero duro, cilíndricos, formados por dos semicilindros que, unidos, permitan obtener probetas cilíndricas de $15 \mathrm{~cm}$ de diámetro y 30 de altura.

f) Cámara húmeda.

g) Cámara de amasado, materiales secos y aguas de amasado. La temperatura de la cámara de amasado, los materiales secos y el agua de amasado se mantendrá entre $20^{\circ}$ y $27,5^{\circ} \mathrm{C}$ y la humedad relativa en la cámara de amasado no será menor que $50 \%$. La cámara de amasado deberá poder ser utilizada a $23^{\circ} \pm 1^{\circ} \mathrm{C}$ y $100 \%$ h. r. y a $23^{\circ} \pm 1^{\circ} \mathrm{C}$ y $50 \%$ h. r. 


\section{MATERIALES}

Arido: arena silícea de tamaño máximo $17 \mathrm{~mm}$. Sulfato sódico cristalizado; calidad reactivo para análisis.

\section{PREPARACION Y CARACTERISTICAS DEL MORTERO}

El mortero deberá tener una relación $a / c$ (en peso) de 0,51.

El escurrimiento será de $7,50 \mathrm{~cm}$ y el aire ocluido no sobrepasará el $5 \% \pm 1 \%$.

El mortero se mezclará de acuerdo con el procedimiento descrito en la sección 6 del método ASTM C 305.

\section{PREPARACION DE LA DISOLUCION}

Como agresivo se utilizará una disolución acuosa de sulfato sódico, cuya concentración será del $2,1 \%$ de $\mathrm{SO}_{4} \mathrm{Na}_{2}$.

Para cada litro de disolución se disolverán $47,63 \mathrm{~g}$ de $\mathrm{SO}_{4} \mathrm{Na}_{2} \cdot 10 \mathrm{H}_{2} \mathrm{O}$ en agua y se completará el volumen a 1 litro. Es aconsejable contrastar mediante el análisis químico la concentración lograda.

\section{CURADO DE LA PROBETA}

Después de extraer (a las 24 horas del amasado) los cilindros de los moldes, se les mantendrán durante 14 días en un recinto cuya temperatura debe ser $23^{\circ} \pm 1^{\circ} \mathrm{C}$ y su humedad relativa $100 \%$. A continuación se conservarán otros 14 días a la misma temperatura pero con sólo el $50 \%$ de humedad relativa.

\section{\%. ATAQUE DE LAS PROBETAS}

El ensayo consiste en 28 días de inmersión en una disolución al 2,1\% de sulfato sódico $\left(\mathrm{SO}_{4} \mathrm{Na}_{2}\right)$ mantenida a $23^{\circ} \pm 1^{\circ} \mathrm{C}$ y, a continuación, secado durante 24 horas al aire del laboratorio. 


\section{MEDIDA DE LAS DEFORMACIONES}

Al terminar los 28 días de curado (ap. 6) se mide la altura de los cilindros utilizando un comparador capaz de apreciar $0,0025 \mathrm{~mm}$.

Al terminar cada etapa del ataque (ap. 7) se realizan nuevas medidas.

\section{CALCUlOS}

Las diferencias de longitud apreciadas en las medidas descritas en (ap. 8), se refieren a la primera medida realizada (que corresponda al final del curado) y se expresan en tanto por ciento de esta longitud.

\section{CALIFICACION}

Las probetas cuya expansión es del $0,2 \%$, o más, se consideran que no soportan el ensayo y su edad deberá consignarse. 


\section{Método del C. E. R. I. L. H.}

La durabilidad del conglomerante se aprecia a través de la cuantía del hinchamiento que experimentan las probetas de un mortero preparado con él y mantenido en contacto con disoluciones de sulfato sódico y de sulfato magnésico.

\section{MATERIALES}

Arena normalizada RILEM-CEMBUREAU.

Arena de composición igual a la anterior, pero de granos menores de 150 micras.

Sulfato sódico, $\mathrm{Na}_{2} \mathrm{SO}_{4} \cdot 10 \mathrm{H}_{2} \mathrm{O}$ (Reactivo para análisis).

Sulfato magnésico, $\mathrm{MgSO}_{4} \cdot 7 \mathrm{H}_{2} \mathrm{O}$ (Reactivo para análisis).

\section{APARATOS}

a) Moldes: Los usualmente empleados (por ejemplo los mencionados en el "Pliego General de Condiciones para la admisión de conglomerantes en las obras de carácter oficial" B.O.E. mayo de 1964) para las probetas de $4 \times 4 \times 16 \mathrm{~cm}$, acondicionados para que se les pueda colocar sendos vástagos de acero inoxidable en sus caras cuadradas, con el fin de realizar medidas de la dimensión mayor.

b) Utensilios para el amasado y enmoldado de las probetas: Los descritos en el "Pliego" antes mencionado.

c) Comparador: El mencionado en el ya citado "Pliego".

\section{COMPOSICION DEL MORTERO DE ENSAYO}

El mortero utilizado en la preparación de las probetas tendrá la composición siguiente:

$1.450 \mathrm{~g}$ de arena RILEM-CEMBUREAU.

$225 \mathrm{~g}$ de arena con tamaño máximo de granos de 150 micras.

$225 \mathrm{~g}$ de conglomerante.

$225 \mathrm{ml}$ de agua. 


\section{PREPARACION DE LAS DISOLUCIONES Y DEL MORTERO}

a) Las disoluciones se preparan disolviendo en agua destilada la cantidad necesaria de la sal respectiva para obtener una concentración del $5 \%$ de sal anhidra $\left(\mathrm{Na}_{2} \mathrm{SO}_{4}\right.$ y $\left.\mathrm{MgSO}_{4}\right)$.

Se recomienda contrastar analíticamente la concentración obtenida y corregirla si fuese menester.

b) La preparación del mortero se realizará según el mencionado "Pliego"; se considerará como conglomerante la suma del conglomerante y la arena fina.

\section{PREPARACION DE LOS PRISIMAS}

Las probetas prismáticas destinadas al ensayo se prepararán siguiendo las instrucciones contenidas en el apartado 2.6. del mencionado "Pliego".

\section{CURAdo PREvio de LOS PRISMas}

Antes de someterlos al ensayo, se mantendrán los prismas en la cámara húmeda y bajo agua en la forma y durante los plazos fijados en el citado "Pliego".

Al finalizar estos plazos se procederá a medir la longitud de las probetas con el comparador, y el resultado de esta medida se considerará como longitud de referencia de las probetas para mediciones posteriores.

\section{\%. MODO DE REALIZAR EL ENSAYO DE DURABILIDAD}

En la forma antes expuesta, se preparan nueve prismas; de éstos, una vez curados y medidos, se conservarán:

- Tres en agua potable (totalmente sumergidos).

- Tres, totalmente sumergidos, en la disolución de sulfato sódico.

- Tres, totalmente sumergidos, en la disolución de sulfato magnésico.

Al finalizar cada uno de los plazos previamente convenidos (por ejemplo 7, 28, 60, 90, .. etc. días) se tomará nota de las alteraciones que presenten las probetas (fisuras, astillamientos, etc.).

Al mismo tiempo se las medirá; las variaciones de longitud se referirán a la longitud inicial considerada como 100 y se compararán con las observadas en las probetas conservadas en agua.

Como información complementaria, se fotografiarán las probetas. 


\section{Método para ensayar la sulfato-durabildad de los conglomerantes}

Dr. F. M. LEA

La susceptibilidad al ataque de los sulfatos, se aprecia a través de la cuantía que alcanza la expansión experimentada por barras cilíndricas de mortero, sumergidas en disoluciones de sulfatos. La expansión se mide por las variaciones experimentadas por la mayor dimensión de las barras.

\section{APARATOS Y MOLDES}

1.1. Amasadora: Según se describe en el párrafo 2.6.1.3. del "Pliego de Condiciones para la recepción de conglomerantes hidráulicos - PCCH-64" del Instituto Eduardo Torroja. B.O.E. n.os 109 y 222 del año 1964.

1.2. Moldes: De acero duro, construido de manera que se puedan obtener probetas cilíndricas, con 2,5 cm de diámetro y $12,7 \mathrm{~cm}$ de altura; y cerrados por una de las bases para poder colocarlos con la dimensión mayor en dirección vertical y llenarlos por el extremo o base abierto.

1.3. Indices: En acero inoxidable utilizables con el comparador que se tenga a disposición.

1.4. Comparador: Cualquier modelo que permita medir la longitud de los prismas (distancia entre índices) apreciando 25 micras, (1/10.000 pulgada).

\section{MATERIALES}

2.1. Arena normalizada: Puede ser, en España, la prescrita en el párrafo 2.6.1.1. del Pliego de Condiciones publicado en el B.O.E. números 109 y 222 del año 1964.

2.2. Sulfatos para las disoluciones agresivas: Los que deseen ensayarse deberán pertenecer a la categoría de "reactivos para análisis".

2.3. Mástique para fijar los índices: Cualquiera que resista, sin variaciones de volumen el influjo de las disoluciones agresivas (por ejemplo el BUTAFIX).

\section{PREPARACION DEL MORTERO}

El mortero se amasará según se prescribe en el Pliego español ya citado, párrafo 2.6.1.5. 


\section{PREPARACION DE LAS DISOLUCIONES AGRESIVAS}

Las disoluciones acuosas destinadas al ataque del hormigón se prepararán con agua desionizada, según la técnica usual en los laboratorios químicos. Las concentraciones serán fijadas al programar el ensayo; pero es conveniente comprobarlas mediante el análisis químico antes de utilizarlas.

\section{LLENADO DE LOS MOLDES}

Los moldes, una vez montados y con las juntas revestidas exteriormente con algún mástique que asegure su estanqueidad, se engrasarán ligeramente en su interior, preferentemente con aceite de tocino.

El mortero se verterá dentro del molde en tres capas sucesivas procurando compactarlas por picado.

\section{CURADO PREVIO}

El mortero se mantendrá dentro de los moldes por lo menos durante 24 horas o hasta que su consistencia permita la extración del cilindro sin que sufra deformaciones.

Los moldes llenos se conservarán en cámara húmeda a $23^{\circ} \pm 2^{\circ} \mathrm{C}$ y $100 \%$ de humedad relativa.

Después de extraer los moldes de mortero, se conservarán en posición horizontal durante 29 días en agua desionizada, cambiándolos de generatriz para evitar una deformación.

\section{\%. FIJACION DE LOS INDICES Y MEDICION INICIAL}

Al terminar el curado previo se fijarán los índices en los extremos del cilindro, y cuando el mástique haya adquirido la necesaria dureza (en cámara húmeda) se realizará una medida con el comparador. Este valor se considera como el de referencia para cada probeta.

\section{ATAQUE DEL MORTERO}

Se colocarán las probetas en el interior de las disoluciones agresivas, las cuales se renovarán semanalmente durante los 3 primeros meses y mensualmente después. Cada vez que se renueve el líquido es conveniente realizar una medida de la longitud de las probetas, previamente enjugadas con un paño húmedo.

\section{CAlCulos}

La longitud de las probetas, apreciada con el comparador, se referirá a la medida inicial y se expresará como un tanto por ciento de dicha dimensión. 


\title{
Método de ensayar la resistencia de los cementos a los sulfatos según la Asociación Inglesa de Fabricantes de Cemento
}

\begin{abstract}
A.P.C.M.
La durabilidad se ensaya en prismas de mortero mantenidos en contacto con disoluciones de sulfatos. A intervalos, programados, de tiempo se mide la variación de longitud.
\end{abstract}

\section{MATERIALES}

Moldes: Los moldes utilizados son los normalizados para el ensayo de la expansión del cemento portland en autoclave.

Pisón de acero, con una superficie de apisonado de $5 \mathrm{~cm}^{2}$.

Arena silícea, que no deje residuo sobre el tamiz de 853 micras y deje un residuo del $10 \%$ ponderal sobre el tamiz de 600 micras.

\section{PREPARACION DEL MORTERO}

La mezcla consiste en una parte en peso de cemento y cuatro de arena, que se amasan con 0,6 partes, en peso, de agua.

\section{PREPARACION DE LAS PROBETAS}

Los moldes se llenan en dos capas; cada una de ellas se compacta con un pisón de acero de $5 \mathrm{~cm}^{2}$ de sección. El mortero se compacta en las esquinas alrededor de los índices de acero inoxidable y a lo largo de la superficie del molde, con el pisón hasta obtener una probeta homogénea. Después que la capa superior se ha compactado, el exceso de mortero se aparta y la superficie se iguala con unos golpes de paletín.

\section{CURADO Y CONSERVACION}

Se cubren las probetas contenidas en los moldes con una lámina de goma y se colocan en la cámara húmeda durante $24 \pm 1 / 2$ horas (h.r. $95 \%$ y $18,9^{\circ} \pm 1,7^{\circ} \mathrm{C}$ ).

Finalizado el plazo, se extraen las probetas de los moldes y se les coloca en agua (mantenida a $18,9^{\circ} \pm 1,7^{\circ} \mathrm{C}$ ) durante $1 / 2$ hora antes de medir por primera vez su longitud. 
Las probetas se mantienen en agua durante 6 días antes de colocarlas en las disoluciones agresivas. Las disoluciones se renuevan mensualmente.

Las probetas se conservan colocadas horizontalmente sobre varillas de cristal en forma que el líquido pueda llegar fácilmente a toda la superficie.

\section{MEDICIONES}

Los cambios de longitud se aprecian mediante un comparador tal como se describe en B.S. $1881: 1952$ Parte 16, párrafo 118 a. (*).

Antes de efectuar la medida, se enjugan las probetas con un paño húmedo. La primera medición se realiza a las 24 ó 25 horas contadas desde el momento que se adicionó el agua a la mezcla. Las mediciones siguientes tienen lugar a los 7 días después y a intervalos regulares hasta que se produce la destrucción de las probetas.

Las diferencias entre la longitud de las probetas a 1 día y al finalizar los plazos siguientes se calcula hasta la centésima por ciento $(0,01 \%)$ de la longitud "efectiva", y se le considera como la expansión de la muestra en ese intervalo.

(Continuará)

(*) Se puede utilizar el descrito en el Pliego español 1964 B.O.E. n.os 109 y 222. 